\title{
The soluble transferrin receptor (TfR)-F-Index is not applicable as a test for iron status in patients with chronic lymphocytic leukemia
}

\author{
Georgia Metzgeroth", Melanie Kripp", Nadine \\ Müller ${ }^{1}$, Beate Schultheis ${ }^{1}$, Karin Bonatz ${ }^{1}$, \\ Christoph Walz ${ }^{1}$, Alexandra Dorn-Beineke ${ }^{2}$ \\ and Jan Hastka ${ }^{1, *}$ \\ ${ }^{1}$ III. Medizinische Klinik, Universitätsmedizin \\ Mannheim, Universität Heidelberg, Mannheim, \\ Germany \\ ${ }^{2}$ Institut für Klinische Chemie, Universitätsmedizin \\ Mannheim, Universität Heidelberg, Mannheim, \\ Germany
}

\begin{abstract}
Background: The soluble transferrin receptor ( $\mathrm{TTRR}$ ) is established as a test for iron deficiency (ID). In chronic lymphocytic leukemia (CLL), sTfR is not reliable for screening for ID as the latter is strongly dependent on tumor burden.

Methods: We investigated whether the influence of the tumor load can be excluded or minimized using the sTfR/log ferritin ratio (TfR-F-Index) and the C-reactive protein (CRP)-adjusted TfR-F-Index in 87 patients with CLL. sTfR was measured nephelometrically (normal: $0.81-1.75 \mathrm{mg} / \mathrm{L}$ ). A cut-off value of 1.5 for the TfR-F-Index and 0.8 for the CRP-adjusted TfR-F-Index, in patients with a CRP $>5 \mathrm{mg} / \mathrm{L}$, was used.

Results: All Binet A patients had normal sTfR values $(1.34 \pm 0.2 \mathrm{mg} / \mathrm{L})$, TfR-F-Index $(0.67 \pm 0.2)$ and a CRPadjusted TfR-F-Index. In Binet $B$ and $C$, sTfR and the TfR-F-Index were significantly increased compared to Binet A patients $(p<0.0001)$. The differences between Binet $B$ and $C$ were not significant. sTfR was increased in $85 \%$, TfR-F-Index in $46 \%$ and the CRP-adjusted TfRF-Index in $54 \%$ of the Binet B patients, in Binet C patients, $80 \%, 50 \%$ and $60 \%$ showed increases, respectively. sTfR and the TfR-F-Index decreased or even normalized following successful treatment.

Conclusions: Similar to sTfR, the TfR-F-Index is strongly associated with tumor burden in patients with CLL. Thus, these parameters do not allow for a reliable diagnosis of ID in this patient group.

Clin Chem Lab Med 2009;47:1291-5.
\end{abstract}

Keywords: chronic lymphocytic leukemia; soluble transferrin receptor; sTfR/log ferritin ratio index.

\footnotetext{
*Corresponding author: Prof. Dr. Jan Hastka, III. Medizinische Klinik, Universitätsmedizin Mannheim, Theodor-Kutzer-Ufer 1-3, 68167 Mannheim, Germany Phone: + 49 (0) 621/383-4115, Fax: + 49 (0) 621/383-4201, E-mail: jan.hastka@umm.de Received April 7, 2009; accepted June 30, 2009; previously published online September 11, 2009
}

\section{Introduction}

Iron deficiency (ID) represents the most common deficiency disease in human beings, and constitutes one of the main factors for anemia in malignancy (1). The clinical diagnosis of ID is based primarily on laboratory parameters, particularly the standard tests for ferritin, transferrin saturation and red cell indices. Recently, newer tests for ID have been established, including measurement of the percentage of hypochromic erythrocytes (HYPO), reticulocyte hemoglobin ( $\mathrm{CHr}$ ), erythrocytic zinc protoporphyrin (ZPP) and serum concentrations of the soluble transferrin receptor (sTfR). These tests are of particular clinical interest as they directly reflect iron supply for erythropoiesis (2-6). sTfR has a significant status within this series of tests because real and functional ID in anemia of chronic disorders can be reliably distinguished $(2,3)$.

However, the diagnostic importance of sTfR as a screening parameter for ID is lessened by the fact that sTfR concentrations are also dependent on erythropoietic activity (2). It is increased not only in ID, but also in diseases with increased erythropoesis $(2,7,8)$. The use of sTfR in lymphoproliferative disorders is particularly tricky, especially in patients with chronic lymphocytic leukemia (CLL). A recent publication suggested that STfR does not reflect transferrin receptor density on tumor cells but rather indicates tumor burden in this patient group (9). Independently of iron status and erythropoetic activity, sTfR values are significantly increased in individuals with large tumor mass, and normalize following successful treatment (9).

To increase the diagnostic sensitivity and specificity for sTfR in detecting ID, the sTfR/log ferritin ratio (TfRF-Index), the ratio of serum concentration of $s T f R$ $(\mathrm{mg} / \mathrm{L})$ and logarithm of the serum ferritin value $(\mu \mathrm{g} / \mathrm{L})$, was introduced $(3,7,10-13)$. In comparison to single measurements of sTfR concentrations, the combination of these two parameters as an indicator of iron stores is considered to be more valuable $(3,12)$. Further diagnostic improvement has been attributed to determination of the C-reactive protein (CRP)-adjusted TfR-F-Index (3, 7, 10-13), where the influence of inflammation on the acute phase reactant ferritin is corrected by CRP (4). With normal CRP values, a TfR-F-Index $>1.5$ defines ID, while for CRP values $>5 \mathrm{mg} / \mathrm{L}$, a cut-off value of $>0.8$ is considered to be suggestive of ID (4).

The goal of the present study was to investigate whether the diagnostic improvement of sTfR achieved by calculation of the TfR-F-Index and the CRP-adjusted TfR-F-Index, is applicable in patients with CLL, and 
in particular if the influence of tumor load can be minimized or even excluded.

\section{Patients and methods}

The study was performed between 2000 and 2008 in the III. Medizinischen Klinik, Universitätsmedizin Mannheim of the University of Heidelberg. Only previously untreated patients with CLL were enrolled and studied. Twelve of these patients received fludarabine-based therapy and were monitored regularly. The diagnosis was based on the National Cancer Institute Criteria (14), including immunophenotyping and bone marrow examination. All patients were staged according to the Binet classification (Table 1) (15). Patients with ID ( $n=16)$, hemolysis and autoimmune thrombocytopenia $(n=11)$ were excluded. In all cases, the presence of ID was excluded by Prussian blue staining of the bone marrow and measurement of ferritin ( $<35 \mu \mathrm{g} / \mathrm{L}$ for males, $<25 \mu \mathrm{g} / \mathrm{L}$ for females).

In addition to determination of serum concentrations of the sTfR, ferritin and the TfR-F-Index, we also obtained complete blood counts including hemoglobin $(\mathrm{Hb})$, platelet counts, white blood cell (WBC) counts and differential counts, as well as further parameters of hemolysis, such as reticulocyte numbers, Coombs-test, haptoglobin, bilirubin and lactate dehydrogenase (LDH) were determined at baseline, and then at regular intervals during the course of the study. In Binet $C$ patients, autoimmune thrombocytopenia was excluded by the determination of platelet associated autoantibodies and bone marrow examination. The measurements of $\mathrm{Hb}$ (reference ranges: $130-170 \mathrm{~g} / \mathrm{L}$ for men, 120-160 g/L for women), mean corpuscular volume (MCV) (reference range: $80-96 \mathrm{fL}$ ), WBC (reference range: $4.2-$ $10.2 \times 10^{9} / \mathrm{L}$ ) and platelet counts (reference ranges: 145$348 \times 10^{9} / \mathrm{L}$ for men, $165-387 \times 10^{9} / \mathrm{L}$ for women) were performed using an automated cell counter (Model CC-180; Sysmex, Kobe, Japan). Serum ferritin (normal value: 35-310 $\mu \mathrm{g} / \mathrm{L}$ for men, $25-210 \mu \mathrm{g} / \mathrm{L}$ for women) was measured using the Access Ferritin immunoassay (Beckman-Coulter, Krefeld, Germany), haptoglobin (normal value: $0.3-2.0 \mathrm{~g} / \mathrm{L}$ ) by the Haptoglobin-Kit (Sentinel Diagnostics, Milan, Italy), bilirubin (normal value: $2-21 \mathrm{mmol} / \mathrm{L}$ ) and $\mathrm{LDH}$ (normal value: 0-248 U/L) were measured with a multi-analysis instrument (SMAC II, Technicon, Tarrytown, NY, USA). Measurement of sTfR (normal range: $0.81-1.75 \mathrm{mg} / \mathrm{L}$ ) was performed using the latex enhanced N Latex STfR assay (Dade Behring, Marburg, Germany). The TfR-F-Index was calculated as the ratio of $\operatorname{sTfR}(\mathrm{mg} / \mathrm{L}$ ) to the logarithm of ferritin $(\mu \mathrm{g} / \mathrm{L}$ ) (normal: $<1.5$ ). For the CRP-adjusted TfR-F-Index, CRP was measured using a turbidimetric high sensitivity assay (Dimension RxL, Fa. Siemens; normal range: $0-5 \mathrm{mg} / \mathrm{L}$ ). For normal CRP concentrations, a TfR-F-Index $>1.5$ defines ID, for CRP $>5$ $\mathrm{mg} / \mathrm{L}$, a cut-off of $>0.8$ was used, as proposed by Thomas and Thomas (4).

Data obtained in our previous study on 43 healthy individuals without ID (mean sTfR: $1.18 \mathrm{mg} / \mathrm{L} \pm 0.2$, range 0.91-1.71; TfR-F-Index: $0.57 \pm 0.12$, range $0.41-0.92$ ) were used as a reference for the statistical analysis (16).

To exclude chemotherapy-related effects on erythropoesis, sTfR, ferritin and the TfR-F-Index, the determination of these parameters were performed 2-4 weeks after the last course of therapy.

Tumor load was assessed in all patients at baseline and then regularly during the course of the study by sonography, evaluating the number of involved lymph node regions and lymph node size. For statistical analysis, the maximal longitudinal diameter of the largest lymph node measured by sonography (Philips iU22, DA Best, Netherlands) was used.
Statistical analyses were performed using GraphPad Prism statistical package for Windows (Version 4.0, GraphPad Software Inc, San Diego, CA, USA). The non-parametric two-tailed Mann-Whitney test was used to determine significances between groups. Differences between frequencies were evaluated for significance using the $\chi^{2}$-test. A $p<0.05$ was considered for statistical significance.

\section{Ethics}

The study was conducted in accordance with the Declaration of Helsinki 1975, as revised in 1983, and approved by the Institutional Review Board. The study did not influence in any way the management of patients, as all the tests used in the study, including bone marrow examination, are routinely performed in all patients with CLL at the time of initial presentation. All patients gave written informed consent.

\section{Results}

We enrolled 87 untreated patients with CLL $(51$ males, 36 females; median age 66 years, range $28-88$ years) into the study. The laboratory data obtained from these patients, subdivided according to their Binet stage, are summarized in Table 1. The individual TfRF-Index for all participants, subdivided according to Binet, are depicted in Figure 1.

Thirty-eight participants (22 males, 16 females; median age 68 years, range 39-87 years) were classified as Binet A. All Binet A patients showed normal sTfR concentrations (mean sTfR $1.34 \pm 0.2 \mathrm{mg} / \mathrm{L}$, range 0.71-1.75), TfR-F-Index $(0.67 \pm 0.2$, range $0.29-1.06)$ and a CRP-adjusted TfR-F-Index (Table 1). When compared to the healthy reference group without ID (16), both the mean sTfR $(p<0.0001)$ and the mean TfR-FIndex $(p=0.017)$ were significantly increased.

Thirty-nine patients (23 males, 16 females, median age 72 years, range $28-86$ years) were staged as Binet $B$. The WBC counts were significantly higher $(p=0.02)$ and the mean lymph node size was significantly greater $(p<0.005)$ than in Binet $A$ individuals (Table 1).

The mean sTfR concentration in Binet B patients was significantly higher when compared to Binet $A$ patients (3.16 $\pm 1.7 \mathrm{mg} / \mathrm{L}$, range $1.3-9.5 ; \mathrm{p}<0.0001)$. The sTfR concentration was increased in $33(85 \%)$ of Binet B patients.

Calculating the TfR-F-Index in the Binet B group, the values were significantly higher when compared to the Binet $A$ individuals $(1.57 \pm 0.8$, range $0.43-4.71$; $\mathrm{p}<0.0001$ ). Normal values were observed in 21 of the Binet B patients; in 18 individuals (46\%), the TfR-FIndex was greater than the cut-off value of 1.5 . The CRP-adjusted TfR-F-Index was increased in 21 (54\%) Binet B patients.

Ten patients $(7$ males, 3 females, median age 64 years, range $46-80$ years) were classified as Binet C. The mean WBC count and the mean maximal lymph node size were significantly higher compared to Binet $A(p=0.02)$. However, there was no significant difference in these parameters when comparing Binet $B$ and $C$ individuals (Table 1 ). 
The mean sTfR concentration in Binet $C$ patients was significantly increased when compared to Binet A patients $(3.67 \pm 2.3 \mathrm{mg} / \mathrm{L}$, range1.6-7.9, $\mathrm{p}<0.0001)$, but did not significantly differ compared to Binet B. Individual sTfR concentrations were increased in eight of $10(80 \%)$ Binet C individuals.

The mean TfR-F-Index was significantly higher compared to Binet $A(2.10 \pm 1.4$; range 0.84-4.80, $\mathrm{p}<0.0001)$ and did not differ compared to Binet $B$ patients. Five patients $(50 \%)$ of the Binet $C$ group showed a TfR-F-Index above 1.5. Six Binet $C$ patients (60\%) showed an abnormal CRP-adjusted TfR-F-Index.

There were no significant differences between the Binet stages with respect to ferritin concentration and transferrin saturation.

Considering all 87 enrolled CLL patients, the sTfR concentration was above the upper reference limit of $1.75 \mathrm{mg} / \mathrm{L}$ in 41 (47\%) patients. The TfR-F-Index, with a threshold value of 1.5 , was increased in $23(26 \%)$ individuals, and the CRP-adjusted TfR-F-Index was abnormal in 27 (31\%) patients.
When considering patients with three or more lymph node regions having significant tumor mass, 45 patients were affected (Binet $A, n=0$; Binet $B$, $\mathrm{n}=39$; Binet $C, \mathrm{n}=6$ ). $\mathrm{sTfR}$ was increased in $38 / 45$ patients, the TfR-F-Index in 23/45 patients and the CRP-adjusted TfR-F-Index in 25/45 patients. Thus, the diagnostic accuracy for the detection of significant tumor load by an increased sTfR, TfR-F-Index and CRP-adjusted TfR-F-Index in CLL patients was 89\%, $74 \%$ and $75 \%$, with a sensitivity of $84 \%, 51 \%$ and $56 \%$ and a specificity of $93 \%, 98 \%$ and $95 \%$, respectively.

In CLL patients with anemia (Binet $A, n=4$; Binet $B$, $\mathrm{n}=11$; Binet $C, \mathrm{n}=5$ ), showing a $\mathrm{Hb}$ level $<130$ $\mathrm{g} / \mathrm{L}$ in men and $<120 \mathrm{~g} / \mathrm{L}$ in women, the sTfR was increased in 14 patients $(70 \%)$, and the TfR-F-Index was increased in seven patients (35\%). Considering the cut-off value of 0.8 for patients with a CRP $>5$ $\mathrm{mg} / \mathrm{L}$, the CRP-adjusted TfR-F-Index was increased in nine of 20 patients (45\%). No significant differences in sTfR and the TfR-F-Index were observed between patients with and without anemia.

Table 1 Characteristics of all the participants, subdivided according to Binet stage.

\begin{tabular}{|c|c|c|c|}
\hline Classification criteria & Binet A & Binet $B$ & Binet C \\
\hline Lymphadenopathy & $<3$ regions & $\geq 3$ regions & Irrelevant \\
\hline $\mathrm{Hb}, \mathrm{g} / \mathrm{L}$ & $>100$ & $>100$ & $\leq 100$ \\
\hline Platelet count, $\times 10^{9} / \mathrm{L}$ & Normal & Normal & $<100$ \\
\hline \multicolumn{4}{|l|}{ Results } \\
\hline n (men/women) & $38(22 / 16)$ & $39(23 / 16)$ & $10(7 / 3)$ \\
\hline \multicolumn{4}{|l|}{ WBC } \\
\hline \multirow[t]{3}{*}[4.2-10.2\times10^{9}/\mathrm{L}]{} & $21.8 \pm 19.6$ & $43.3 \pm 44.1$ & $49.8 \pm 30.2$ \\
\hline & $(6.2-133.0)$ & $(3.5-207)$ & $(10.5-99.9)$ \\
\hline & & $\{A$ vs. $B, 0.02\}$ & $\{B$ vs. $C$, n.s. $\}$ \\
\hline \multicolumn{4}{|l|}{$\mathrm{Hb}$} \\
\hline [Men: 130-170 g/L] & $139 \pm 10.2$ & $133 \pm 14.3$ & $126 \pm 15.0$ \\
\hline \multirow[t]{2}{*}{ [Women: $120-160 \mathrm{~g} / \mathrm{L}$ ] } & $(115-195)$ & $(104-163)$ & $(92-144)$ \\
\hline & & $\{A$ vs. $B$, n.s. $\}$ & $\{B$ vs. $C$, n.s. $\}$ \\
\hline \multicolumn{4}{|l|}{ Platelet count } \\
\hline [Men: 145-348×109/L] & $222 \pm 65$ & $190 \pm 55$ & $81 \pm 10$ \\
\hline \multirow{2}{*}{ [Women: $\left.165-387 \times 10^{9} / \mathrm{L}\right]$} & $(123-457)$ & $(108-325)$ & $(70-95)$ \\
\hline & & $\{A$ vs. $B$, n.s. $\}$ & $\{B$ vs. $C, 0.0001\}$ \\
\hline \multicolumn{4}{|l|}{ Reticulocyte count } \\
\hline \multirow[t]{3}{*}{$5-15 \%$ o } & $11 \pm 6$ & $12 \pm 6$ & $12 \pm 5$ \\
\hline & $(1-18)$ & $(4-17)$ & $(7-18)$ \\
\hline & & $\{A$ vs. $B$, n.s. $\}$ & $\{B$ vs. $C$, n.s. $\}$ \\
\hline \multicolumn{4}{|l|}{ Ferritin } \\
\hline [Men: 35-310 $\mu \mathrm{g} / \mathrm{L}]$ & $156 \pm 143$ & $160 \pm 194$ & $76 \pm 54$ \\
\hline \multirow[t]{2}{*}{ [Women: $25-210 \mu \mathrm{g} / \mathrm{L}]$} & $(25-728)$ & $(25-1160)$ & $(25-187)$ \\
\hline & & $\{A$ vs. $B$, n.s. $\}$ & $\{B$ vs. $C$, n.s. $\}$ \\
\hline \multirow[t]{3}{*}{ Lymph node size ${ }^{a}, \mathrm{~cm}$} & $1.4 \pm 0.5$ & $3.9 \pm 1.9$ & $2.8 \pm 1.4$ \\
\hline & $(0.7-3.0)$ & $(1.6-9.0)$ & $(1.0-5.8)$ \\
\hline & & $\{A$ vs. $B,<0.005\}$ & $\{B$ vs. $C$, n.s. $\}$ \\
\hline \multicolumn{4}{|l|}{ sTfR } \\
\hline \multirow[t]{3}{*}[0.81-1.75\mathrm{mg}/\mathrm{L}]{} & $1.34 \pm 0.2$ & $3.16 \pm 1.7$ & $3.67 \pm 2.3$ \\
\hline & $(0.71-1.75)$ & $(1.3-9.5)$ & $(1.6-7.9)$ \\
\hline & & $\{A$ vs. $B,<0.0001\}$ & $\{B$ vs. $C$, n.s. $\}$ \\
\hline \multirow[t]{3}{*}{ TfR-F-Index } & $0.67 \pm 0.2$ & $1.57 \pm 0.8$ & $2.10 \pm 1.4$ \\
\hline & $(0.29-1.06)$ & $(0.43-4.71)$ & $(0.84-4.80)$ \\
\hline & & $\{A$ vs. $B,<0.0001\}$ & $\{B$ vs. $C$, n.s. $\}$ \\
\hline \multicolumn{4}{|l|}{ TfR-F-Index, CRP-adjusted } \\
\hline [Increased/normal, (\% increased)] & $0 / 38(0 \%)$ & $21 / 18(54 \%)$ & $6 / 4(60 \%)$ \\
\hline
\end{tabular}

The values are mean \pm SD (and range). Values in square brackets are reference values, values in curly brackets are $p$-values; ${ }^{a}$ mean maximal lymph node size in cm; n.s., non-significant. Hb, hemoglobin; WBC, white blood cell; sTfR, soluble transferrin receptor; TfR-F-Index, sTfR/log ferritin ratio; CRP, C-reactive protein; SD, standard deviation. 


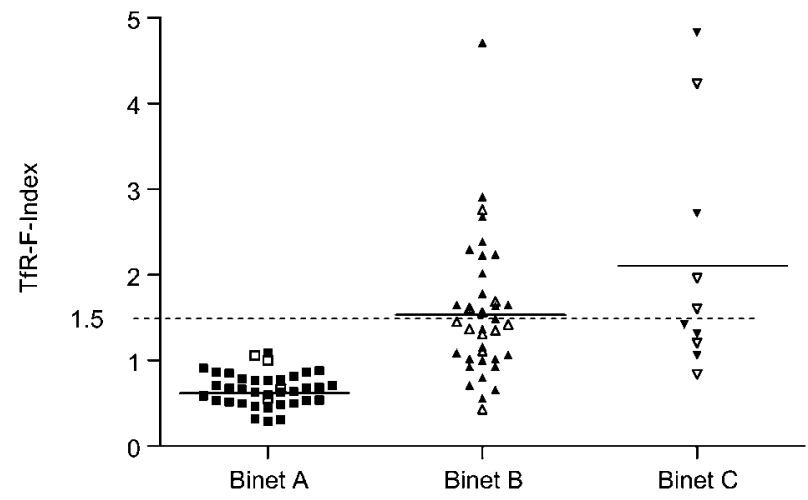

Figure 1 Individual TfR-F-Index and mean TfR-F-Index ( - ) for all CLL patients, subdivided according to their Binet stage.

All Binet A patients had a normal TfR-F-Index. In Binet B patients, the mean TfR-F-Index was significantly increased $(p<0.0001)$, the further increase in Binet $C$ patients was not significant. The dotted line represents the threshold value of 1.5 indicating the onset of ID in patients with a CRP value below $5 \mathrm{mg} / \mathrm{L}$. The empty marks represent the 20 anemic patients $(\mathrm{Hb}$ in females $<120 \mathrm{~g} / \mathrm{L}$, in males $<130 \mathrm{~g} / \mathrm{L})$.

Twelve individuals received up to six cycles (mean 4 , range 4-6) of fludarabine-based therapy. All responded to treatment with complete hematological remission and partial or complete disappearance of lymph node enlargement. All these patients had an increased TfR-F-Index and an increased sTfR serum concentration before the initiation of treatment.

The high pre-treatment sTfR values $(7.18 \pm 2.1 \mathrm{mg} / \mathrm{L}$, range 4.3-9.5) and TfR-F-Index (4.09 \pm 2.0 , range 2.2-9.5) progressively declined to near normal values following four cycles of therapy (mean TfR-F-Index $1.02 \pm 0.3$, range $0.5-1.7, \mathrm{p}<0.0001$, and mean $\mathrm{STfR}$ $2.10 \pm 1.0 \mathrm{mg} / \mathrm{L}$, range $1.2-4.7, \mathrm{p}<0.0001$, respectively), as shown in Figure 2.

The decline of the TfR-F-Index in responders began immediately following the start of therapy and was statistically significant compared to pre-treatment values after the second course of the treatment $(4.09$ vs. $2.13, p=0.002$ ). The decrease continued during the following courses, reaching normal TfR-F-Index by the end of therapy in all 11 patients with complete clinical remission. The remaining patient achieved only partial remission after four cycles of chemotherapy, and the TfR-F-Index remained increased with an end of treatment value of 1.69 (Figure 2).

\section{Discussion}

Our current data suggest that calculation of the TfRF-Index does not increase the reliability of the STfR concentration in the assessment of iron status in patients with CLL. Similar to sTfR, the TfR-F-Index depends on tumor load, and shows increased values in advanced CLL despite the lack of ID or increased erythropoesis.

The TfR-F-Index was introduced to increase the diagnostic sensitivity and specificity of STfR in the assessment of ID $(3,7,10-13)$. There are several rea-

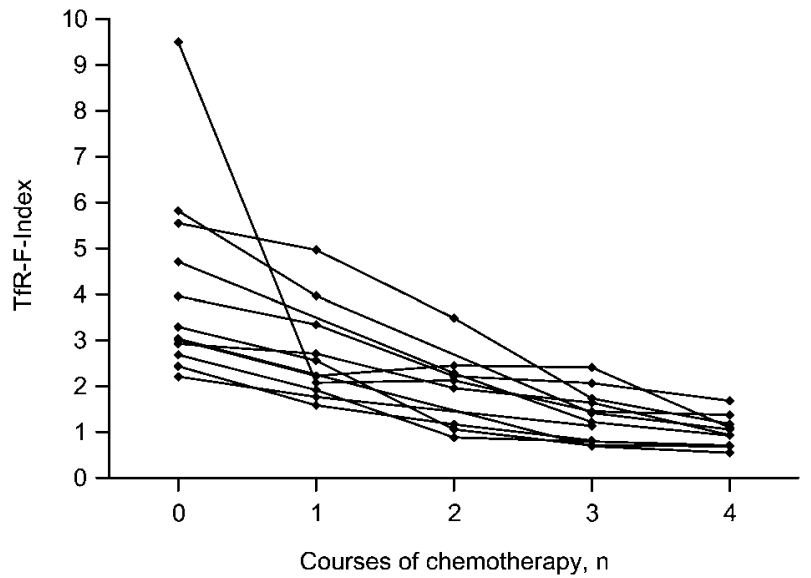

Figure 2 Individual TfR-F-Index time courses for 12 patients, responding to a fludarabine-based regime that was given every 4 weeks.

In these responders, the application of the fludarabine-based therapy was accompanied by a continuous decline in the TfR-F-Index, which normalized in 11 patients who achieved complete remission. The decline began immediately after initiation of treatment, and was statistically significant after the second course of therapy $(p=0.002)$.

sons for the diagnostic improvement of sTfR for detecting ID using determination of the TfR-F-Index. The increased sensitivity is based on the ability of ferritin to detect deficiencies in storage iron, whereas the sTfR concentration only increases when the iron available for erythropoesis becomes insufficient. Ferritin also contributes to the higher specificity of the TfR-F-Index in comparison to single determinations of sTfR alone, by amplifying the influence of ID and attenuating the "erythropoesis part" of the sTfR concentration. Thus, there was a legitimate hope to exclude or minimize the influence of tumor burden on the sTfR concentration in patients with CLL by combining sTfR and ferritin through the use of the TfR-F-Index.

When compared with sTfR, which was increased in nearly half of the patients, about one quarter of the individuals showed an increased TfR-F-Index at diagnosis. These results show that calculation of the TfRF-Index reduces the influence of tumor load in CLL patients when compared to determination of sTfR concentrations alone. Although the difference was statistically significant $(p=0.01)$, a $26 \%$ false positive rate is not a sound base for detection of ID in these individuals. However, it should be mentioned that increased concentrations of sTfR and the TfR-F-Index were restricted to Binet $B$ and $C$ patients. The sTfR concentration and the TfR-F-Index were normal in all Binet $A$ individuals.

To reduce the influence of inflammation on the acute phase reactant ferritin, an interpretation of the TfR-F-Index based on CRP concentration has been proposed (3, 7, 10-13). The application of this strategy using the CRP-adjusted TfR-F-Index did not improve the results in our study, and even seemed to impair its value. Considering the CRP-adjusted threshold, the number of patients with increased TfR-F-values increased from $23(26 \%)$ to $27(31 \%)$. With this para- 
meter as well, increased values were restricted to CLL patients staged as Binet B or C.

There is no doubt that the observed increase in the TfR-F-Index in patients with CLL - similar to the increase in the STfR concentration - is the result of high tumor burden. ID and increased erythropoesis were carefully excluded. In all cases, ferritin values were above the lower limit of normal, there was no significant difference in mean ferritin concentrations between the Binet stages and there was detectable storage iron in the bone marrow. The dependence of sTfR and TfR-F-Index on tumor burden is evident with the strong correlation between these parameters and Binet classification, which is primarily determined by tumor load. Patients with low tumor burden and fulfilling the Binet A criteria had, without exception, a TfR-F-Index below the threshold value. However, the mean TfR-F-Index was increased when compared to normal individuals.

The increase in tumor load in Binet $B$ and $C$ patients was accompanied by a significant increase in the mean TfR-F-Index and sTfR concentrations. The proportion of patients with increased TfR-F-Index increased from $0 \%$ in Binet $A$ to $46 \%(18 / 39)$ in Binet $B$, and $50 \%(5 / 10)$ in Binet $C$ patients. However, the increase compared between Binet B and C was not statistically significant. This finding is not surprising taking into account that Binet $C$ stage is primarily defined by hematological insufficiency, and depends on the tumor load indirectly only.

Similar to sTfR concentrations, the TfR-F-Index did not show only excellent correlation with tumor burden at the time of diagnosis, but also within the course of the disease during therapy. In the 12 patients responding to therapy, all but one patient achieved a normal TfR-F-Index (Figure 2), which was accompanied by significant regression or even disappearance in the lymph nodes. Complete remission was always associated with complete normalization of the TfR-F-Index. These findings further support the close association between the TfR-F-Index and tumor mass in CLL.

One may argue that STfR and the TfR-F-Index are reliable tests for iron in Binet A patients, as both parameters were within the normal range in this group. The same could be proposed for patients who achieve complete remission, which was always accompanied by complete normalization of sTfR and the TfR-FIndex. However, one should be aware that in cases of CLL relapse or disease progression, both parameters increase due to increasing tumor mass (9). This is of particular importance as it was demonstrated that increasing sTfR precedes visible lymph node enlargement by 1-4 months (9). Based on this aspect, the differentiation between ID and disease progression by measurement of these two parameters would be impossible.

In summary, our data demonstrate that the TfR-FIndex, similar to the sTfR serum concentration, is closely associated with tumor load at diagnosis and during the course of the disease in patients with CLL. Therefore, use of these parameters is not appropriate for screening of ID in this patient group.

\section{Conflict of interest disclosure}

The authors declare no competing financial interests.

\section{References}

1. Birgegard G, Aapro MS, Bokemeyer C, Dicato M, Drings P, Hornedo J, et al. Cancer-related anemia: pathogenesis, prevalence and treatment. Oncology 2005;68:3-11.

2. Cook JD, Skikne BS, Baynes RD. Serum transferrin receptor. Annu Rev Med 1993;44:63-74.

3. Punnonen K, Irjala K, Rajamaki A. Serum transferrin receptor and its ratio to serum ferritin in the diagnosis of iron deficiency. Blood 1997;89:1052-7.

4. Thomas C, Thomas L. Biochemical markers and hematologic indices in the diagnosis of functional iron deficiency. Clin Chem 2002;48:1066-76.

5. Hastka J, Lasserre JJ, Schwarzbeck A, Hehlmann R. Central role of zinc protoporphyrin in staging iron deficiency. Clin Chem 1994;40:768-73.

6. Cook JD. Iron-deficiency anaemia. Baillieres Clin Haematol 1994;7:787-804.

7. Punnonen K, Irjala K, Rajamaki A. Iron-deficiency anemia is associated with high concentrations of transferrin receptor in serum. Clin Chem 1994;40:774-6.

8. Beguin $Y$. Soluble transferrin receptor for the evaluation of erythropoiesis and iron status. Clin Chim Acta 2003; 329:9-22.

9. Metzgeroth G, Schultheis B, Kuhn C, Dorn-Beineke A, LaRosee $P$, Hehlmann $R$, et al. The soluble transferrin receptor reflects tumor load in chronic lymphocytic leukemia. Clin Chem Lab Med 2007;45:1313-8.

10. Lee EJ, Oh EJ, Park YJ, Lee KH, Kim BK. Soluble transferrin receptor (sTfR), ferritin, and $s T f R / l o g$ ferritin index in anemic patients with non-hematologic malignancy and chronic inflammation. Clin Chem 2002;48:111821.

11. Punnonen K, Irjala K, Rajamaki A. Serum transferrin receptor, ferritin and TfR-F index in identification of latent iron deficiency. Eur J Haematol 1998;60:135-7.

12. Suominen P, Punnonen K, Rajamaki A, Irjala K. Serum transferrin receptor and transferrin receptor-ferritin index identify healthy subjects with subclinical iron deficits. Blood 1998;92:2934-9.

13. Suominen P, Mottonen T, Rajamaki A, Irjala K. Single values of serum transferrin receptor and transferrin receptor ferritin index can be used to detect true and functional iron deficiency in rheumatoid arthritis patients with anemia. Arthritis Rheum 2000;43:1016-20.

14. Cheson BD, Bennett JM, Grever M, Kay N, Keating MJ, O'Brien S, et al. National Cancer Institute-sponsored Working Group guidelines for chronic Iymphocytic leukemia: revised guidelines for diagnosis and treatment. Blood 1996;87:4990-7.

15. Binet JL, Lepoprier M, Dighiero G, Charron D, d'Athis $P$, Vaugier G, et al. A clinical staging system for chronic Iymphocytic leukemia: prognostic significance. Cancer 1977;40:855-64.

16. Metzgeroth G, Adelberger V, Dorn-Beineke A, Kuhn C, Schatz M, Maywald O, et al. Soluble transferrin receptor and zinc protoporphyrin - competitors or efficient partners? Eur J Haematol 2005;75:309-17. 